\title{
Three-Dimensional Modeling of Self-Organization in DC Glow Microdischarges
}

\author{
P. G. C. Almeida, M. S. Benilov, and M. J. Faria
}

\begin{abstract}
Three-dimensional simulations of self-organization in de glow microdischarges are reported. The results describe a mode with a normal spot and modes with patterns of multiple spots, qualitatively similar to those observed in experiments with microdischarges in xenon.
\end{abstract}

Index Terms-Glow discharge, self-organization, normal discharge.

$\mathbf{I}$ MPORTANT advances have been achieved in selfconsistent modeling of self-organization in gas discharges, such as patterns in DBDs and the normal current density effect in dc glow discharges (e.g., [1] and [2], respectively). The initial steps toward a self-consistent modeling of self-organization in dc glow discharges have been taken in [3] and [4]. It was shown that the simplest self-consistent model of glow discharges admits multiple solutions which exist at the same value of the discharge current. Those of the solutions that are 2-D (axially symmetric) were calculated and found to describe either the normal mode or patterns which appear to be 2-D analogs of patterns observed in microdischarges in Xe [5].

In this paper, simulations of 3-D patterns in dc glow microdischarges are reported for the first time. The model is the same as in [4]; it accounts for only one ion species (molecular ions) and makes use of the local approximation for electron kinetics and transport. Note that results [6] indicate that an account of detailed plasma chemistry and the nonlocality of electron energy does not alter results qualitatively. A steady-state solver of Comsol Multiphysics was employed. (The use of a nonstationary solver would not allow finding and understanding the whole pattern of existing steady-state solutions since such solvers do not compute sections of solutions which are unstable with the power supply circuit being considered.) Information on where to look for multiple solutions and what they are like was obtained by means of bifurcation analysis, similarly to how it was done in [4].

The results reported in this paper refer to a cylindrical discharge vessel with parallel electrodes and a reflecting lateral wall. The pattern of multiple solutions for such vessel is the

Manuscript received December 1, 2010; revised April 12, 2011; accepted April 16, 2011. Date of publication June 13, 2011; date of current version November 9, 2011. This work was supported in part by Project PTDC/FIS/ 68609/2006 of FCT, POCI 2010, and FEDER and in part by the Centro de Ciências Matemáticas of FCT, POCTI-219, and FEDER.

The authors are with the Departamento de Física, Universidade da Madeira, 9000 Funchal, Portugal (e-mail: pedroa@uma.pt; benilov@uma.pt; murjeh@ uma.pt).

Color versions of one or more of the figures in this paper are available online at http://ieeexplore.iee.org.

Digital Object Identifier 10.1109/TPS.2011.2148129 simplest and the easiest to understand [3]. (Note that the effect of neutralization of ions and electrons at the wall is likely to be the same as for 2-D solutions [3], [4].) The radius and height of the vessel are equal to $0.5 \mathrm{~mm}$. The plasma-producing gas is xenon, and its pressure is 30 torr. For these conditions, the problem admits solutions describing 16 different modes of current transfer: a 1-D mode, two 2-D modes, and thirteen 3-D modes. The 1-D mode is well known, and the physics behind it is similar to that described by the classical von Engel and Steenbeck solution. The 2-D modes were reported in [3] and [4].

The CVCs of the 1st, 8th, and 12th 3-D modes are shown in Fig. 1(a) ( $U$ is the discharge voltage and $\langle j\rangle$ is the average axial current density). Also shown is the CVC of the 1-D mode. Each of the 3-D modes exists in a limited range of values of the discharge current and branches off from (or joins) the 1-D mode at two points (bifurcation points): one at low currents and the other one in the vicinity of the point of minimum of the CVC of the 1-D mode. All the bifurcations involving the 1st and 12th modes are subcritical (the 3-D mode branches off "backward," as can be seen in the inset). Both bifurcations involving the 8th mode are supercritical.

Distributions of the current density over the surface of the cathode are shown in Fig. 1(b)-(d). Note that the states corresponding to $\langle j\rangle=2.4,394$, and $339 \mathrm{~A} \cdot \mathrm{m}^{-2}$ in Fig. 1(b) are positioned between the corresponding bifurcation and turning points all the states shown in Fig. 1(d) are positioned between the turning points.

In the vicinity of bifurcation points, the distributions of discharge parameters along the cathode are smooth and governed by the Helmholtz equation, in accordance with the theory [7]. Localized current spots are formed away from the vicinity of the bifurcation points. The first mode exhibits the normal current density effect: There is a large plateau in the corresponding $\mathrm{CVC}$, and the maximum current density on the cathode surface remains virtually unchanged in states on this plateau. The 2 nd and subsequent modes exhibit patterns with multiple spots. In general, this pattern of self-organization corresponds to the theory [7] and is similar to the pattern of self-organization of 2-D modes [4].

The effect of normal current density shown in Fig. 1(a) and (b) corresponds to the experimental observations, and the spot patterns shown in Fig. 1(c) and (d) are qualitatively similar to those observed in microdischarges in Xe [5]. There is a minimum or, respectively, a maximum of the current density at the center of the cathode in most states shown in Fig. 1(c) and (d). This result cannot be obtained by means of bifurcation analysis (there is no extremum at the center in distributions governed by the Helmholtz equation) and is important since 

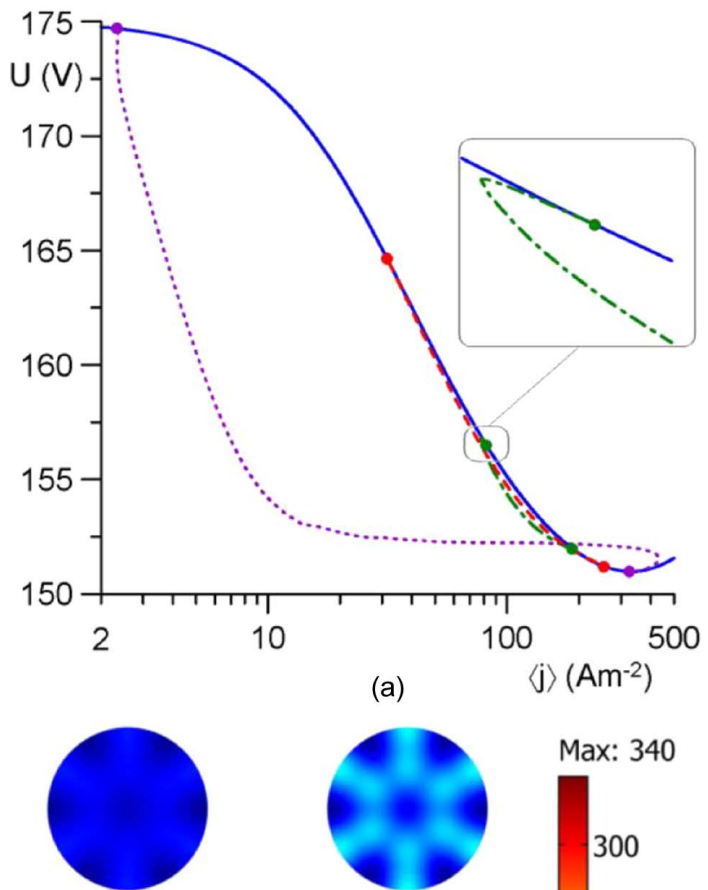

$48 \mathrm{Am}^{-2}$

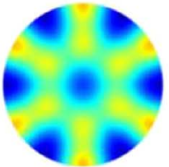

$150 \mathrm{Am}^{-2}$

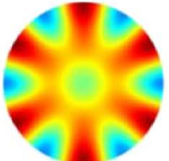

$220 \mathrm{Am}^{-2}$ (a)

$\langle\mathrm{j}\rangle\left(\mathrm{Am}^{-2}\right)$

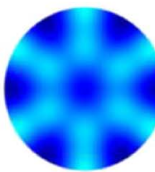

$91 \mathrm{Am}^{-2}$

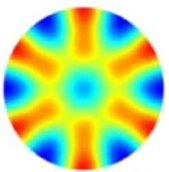

$190 \mathrm{Am}^{-2}$

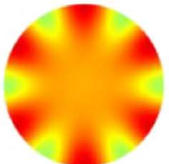

(c)
Max: 340

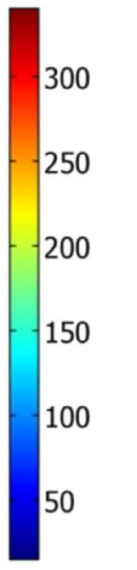

Min: 15.0

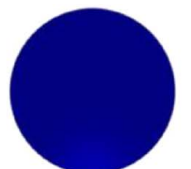

$2.4 \mathrm{Am}^{-2}$

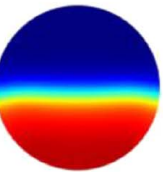

$261 \mathrm{Am}^{-2}$

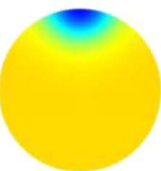

$394 \mathrm{Am}^{-2}$

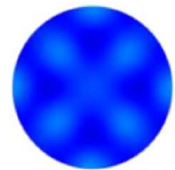

$78 \mathrm{Am}^{-2}$

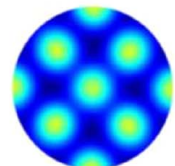

$98 \mathrm{Am}^{-2}$

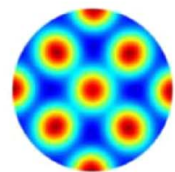

$147 \mathrm{Am}^{-2}$

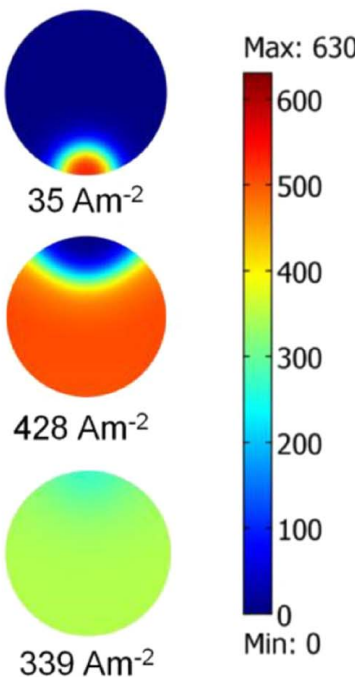

(b)

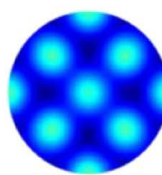

$86 \mathrm{Am}^{-2}$

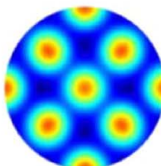

$119 \mathrm{Am}^{-2}$

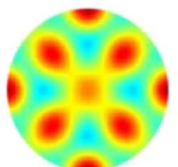

$188 \mathrm{Am}^{-2}$
Max: 290

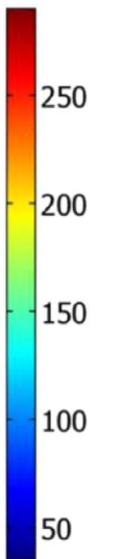

Min: 35.0

Fig. 1. (a) CVCs of the (solid line) 1-D mode and the (dotted line) 1st, (dashed line) 8th, and (dashed-dotted line) 12th 3-D modes. (Circles) Bifurcation points in which 3-D modes branch off from the 1-D mode. (b)-(d) Distributions of current density over the cathode surface in states belonging to 3-D modes. Bars in $\mathrm{A} \cdot \mathrm{m}^{-2}$. (b) $1 \mathrm{st}$ mode. (c) 8 th mode. (d) 12 th mode.

both the patterns with and without spot at the center have been observed in the experiment [5].

\section{ACKNOWLEDGMENT}

The authors P. G. C. Almeida and M. J. Faria would like to thank FCT for the Ph.D. fellowships under Grants SFRH/BD/30598/2006 and SFRH/BD/35883/2007.

\section{REFERENCES}

[1] L. Stollenwerk, S. Amiranashvili, J. P. Boeuf, and H. G. Purwins, "Measurement and 3-D simulation of self-organized filaments in a barrier discharge," Phys. Rev. Lett., vol. 96, no. 25, p. 255001 , Jun. 2006.
[2] R. R. Arslanbekov and V. I. Kolobov, "Two-dimensional simulations of the transition from Townsend to glow discharge and subnormal oscillations," J. Phys. D, Appl. Phys., vol. 36, no. 23, pp. 2986-2994, Dec. 2003.

[3] P. G. C. Almeida, M. S. Benilov, M. D. Cunha, and M. J. Faria, "Analysing bifurcations encountered in numerical modelling of current transfer to cathodes of dc glow and arc discharges," J. Phys. D, Appl. Phys., vol. 42, no. 19 , p. 94010 , Oct. 2009.

[4] P. G. C. Almeida, M. S. Benilov, and M. J. Faria, "Multiple solutions in the theory of dc glow discharges," Plasma Sources Sci. Technol., vol. 19, no. 2, p. 025019 , Apr. 2010.

[5] K. H. Schoenbach, M. Moselhy, and W. Shi, "Self-organization in cathode boundary layer microdischarges," Plasma Sources Sci. Technol., vol. 13, no. 1, pp. 177-185, Feb. 2004

[6] P. G. C. Almeida, M. S. Benilov, and M. J. Faria, "Modelling selforganization in DC glow microdischarges in xenon and argon," in Proc. 63rd GEC and 7th ICRP, Bull. Amer. Phys. Soc., vol. 55, p. 166.

[7] M. S. Benilov, "Theory of structures in near-electrode plasma regions," Phys. Rev. A, vol. 45, no. 8, pp. 5901-5912, Apr. 1992. 